\title{
Radiation pneumonitis in lung cancer treated with volumetric modulated arc therapy
}

\author{
Kan $\mathrm{Wu}^{1 \#}$, Xiao $\mathrm{Xu}^{2 *}$, Xiadong $\mathrm{Li}^{2}$, Jiahao Wang ${ }^{2}$, Lucheng $\mathrm{Zhu}^{2}$, Xueqin Chen ${ }^{1}$, Bing Wang ${ }^{2}$, Minna Zhang $^{2}$, \\ Bing $\mathrm{Xia}^{2}$, Shenglin $\mathrm{Ma}^{1}$
}

${ }^{1}$ Department of Oncology, ${ }^{2}$ Department of Radiation Oncology, The Fourth Clinical Medical College of Zhejiang Chinese Medical University, Hangzhou First People's Hospital, Hangzhou Cancer Hospital, Hangzhou 310006, China

Contributions: (I) Conception and design: K Wu, X Xu, B Xia, S Ma; (II) Administrative support: X Li, X Chen; (III) Provision of study materials or patients: K Wu, X Xu, X Li, J Wang; (IV) Collection and assembly of data: X Xu, M Zhang, B Wang; (V) Data analysis and interpretation: K Wu, L Zhu; (VI) Manuscript writing: All authors; (VII) Final approval of manuscript: All authors.

\#These authors contributed equally to this work.

Correspondence to: Bing Xia. Department of Radiation Oncology, The Fourth Clinical Medical College of Zhejiang Chinese Medical University, Hangzhou First People’s Hospital, Hangzhou Cancer Hospital, No. 261 Huansha Road, Hangzhou 310006, China. Email: xb0918@hotmail.com; Shenglin Ma. Department of Oncology, The Fourth Clinical Medical College of Zhejiang Chinese Medical University, Hangzhou First People's Hospital, Hangzhou Cancer Hospital, No. 261 Huansha Road, Hangzhou 310006, China. Email: mashenglin@medmail.com.cn.

Background: Few studies to date have assessed the incidence of radiation pneumonitis (RP) in lung cancer patients who have been treated with volumetric modulated arc therapy (VMAT). This study is aimed at reporting the RP incidence rate and the risk factors associated with a symptomatic RP in patients with lung cancer treated with VMAT.

Methods: A total of 77 consecutive lung cancer patients treated with VMAT from 2013 through 2015 were reviewed. RP severity was graded according to the Common Terminology Criteria for Adverse Events (CTCEA) v.4. Univariate and multivariate analyses were performed to identify the significant factors associated with RP.

Results: VMAT allowed us to achieve most planning objectives on the target volumes and organs at risk, for PTV $V_{95 \%}=96.8 \% \pm 3.1 \%$, for lung $V_{5}=41.3 \% \pm 8.7 \%, V_{10}=30.0 \% \pm 7.1 \%, V_{20}=20.9 \% \pm 5.7 \%$, for heart $\mathrm{V}_{5}=43.2 \% \pm 29.9 \%$, for esophagus $\mathrm{V}_{60}=8.1 \% \pm 12.9 \%$. The maximum dose of spinal cord was 34.4 $\pm 9.5 \mathrm{~Gy}$. The overall incidence of symptomatic RP (grade $\geq 2$ by CTCAE) was $28.6 \%$ in the entire cohort, and the rate of grade $\geq 3 \mathrm{RP}$ was $11.7 \%$. Based on the multivariate analysis, factors predictive of symptomatic RP included lung volume receiving $\geq 10$ Gy $\left(\mathrm{V}_{10}\right)(\mathrm{P}=0.019)$ and C-reactive protein changing level $(\mathrm{P}=0.013)$.

Conclusions: Our data showed that the incidence rate of RP was acceptable in lung cancer patients treated with VMAT. Additionally, we found that $V_{10}$ might be an important factor for predicting the development of RP when VMAT was used; but this observation needs to be validated in future studies.

Keywords: Lung cancer; volumetric modulated arc therapy; radiation pneumonitis

Submitted Apr 21, 2018. Accepted for publication Nov 20, 2018.

doi: $10.21037 /$ jtd.2018.11.132

View this article at: http://dx.doi.org/10.21037/jtd.2018.11.132

\section{Introduction}

Radiotherapy is an effective treatment modality in lung cancer patients, but the major dose-limiting toxicity effect for the patients undergoing thoracic radiotherapy is radiation pneumonitis (RP). Once symptomatic RP occurs, it typically influences the quality of life and subsequent treatments, possibly leading to poor outcomes. It was reported that the incidence of RP ranges from $17 \%$ to $47 \%$, and the differences in RP risk across the studies might be related to the different criteria for the definition of RP, population 
heterogeneity, and the differences in treatment (1-3).

Recently, the introduction of volumetric modulated arc therapy (VMAT) has provided more conformal target coverage, and is capable of sparing more of the organs at risk (OAR). It has the advantages of a reduced treatment delivery time, and fewer MUs when compared with threedimensional conformal radiation therapy (3D-CRT) and intensity modulated radiation therapy (IMRT) (4-6). However, there is a larger volume of lung cancer patients that received low radiation doses $\left(V_{5}\right.$ and $\left.V_{10}\right)$ when VMAT was delivered (7). A recent study reported that the incidence of symptomatic RP nearly doubled in the VMAT group compared to the IMRT group for non-small cell lung cancer (NSCLC), although this difference was not statistically significant (8). To date, there have been many studies focused on the risks associated with RP for lung cancer patients, treated with 3D-CRT or IMRT $(9,10)$, but fewer publications have reported the RP incidence rate and assessed the risk factors associated with RP in lung cancer patients treated with VMAT. Radiation-induced lung injury is a general term for damage to the lungs which occurs as a result of exposure to ionizing radiation. In general terms, such damage is divided into early inflammatory damage (RP) and later complications of chronic scarring (radiation fibrosis). Therefore, the current article aims to report the $\mathrm{RP}$ incidence in lung cancer patients treated with VMAT to give an idea of the importance in clinical practice. In addition, possible risk factors associated with symptomatic $\mathrm{RP}$ will be introduced to raise any lingering questions.

\section{Methods}

\section{Patients}

After approval by our institutional review board, we reviewed charts of consecutive patients with lung cancer treated at our institution. Patients were considered eligible and were enrolled in this study if they met the following inclusion criteria: (I) thoracic radiotherapy was delivered with VMAT; (II) radiation dose was not less than 45 Gy; (III) information regarding clinical characteristics and treatment planning were available.

From January 2013 through March 2015, thoracic radiotherapy with VMAT was administered in 77 lung cancer patients. Thirty-one patients $(40.3 \%)$ presented with adenocarcinoma, 25 patients $(32.5 \%)$ presented with squamous cell carcinoma, 9 patients $(11.7 \%)$ presented with unspecified NSCLC, and 12 patients (15.6\%) presented with small cell lung cancer. Patients with stage II (3.9\%), III (75.3\%) and IV (20.8\%) lung cancer were recruited into the study, and received adjuvant, definitive and palliative radiotherapy respectively.

Twenty-three patients (29.9\%) were subject to Radiation therapy (RT) in regimens alone. Fifty-four patients were subject to platinum-based chemo-radiotherapy regimens: $39.0 \%$ sequential and $31.2 \%$ concomitant. Concurrent chemotherapy was initiated simultaneously with RT, $1-2$ cycles were concurrently administered with radiotherapy, and 2-3 cycles were administered within 4 weeks of completing the RT. Sequential chemotherapy was initiated 2-4 weeks after RT, 4 cycles were administered, and every 21 days was a cycle. Patients received a median dose of 56 Gy (range, 45-64 Gy) with a median fractionation regimen of $2.0 \mathrm{~Gy}$ (range, 1.8-2.0 Gy) in 28 fractions (range, 25-32 Gy). A total of 52 patients (mostly locally advanced patients) accepted a 4D-CT scan.

For this analysis, relevant information was also recorded. The clinical information included sex, age, performance status (PS) score, smoking history, thoracotomy history, histology, stage, Charlson comorbidity index (recorded as an age-adjusted Charlson Comorbidity Index score for each patient at the time of initial presentation to a radiation oncologist), history of pulmonary disease (included chronic obstructive pulmonary disease and asthma), tumor location, normal lung volume, and chemotherapy regimens. The laboratory results of these patients, include neutrophils (\%), hemoglobin, C-reactive protein (CRP), and lactate dehydrogenase levels examined a week before (Results A) and after radiotherapy (Results B) were collected. In addition, dosimetric parameters from the treatment plans, including the number of arcs, planning target volume (PTV) volume, radiation dose, delivery time and dose volume data of OAR, were also collected (Tables 1 and 2).

\section{Radiation therapy planning}

Patients were immobilized in the supine position with their arms over their head, using a thermoplastic body frame. A free breathing contrast-enhanced helical CT scan with 5 -mm slice thickness was performed in the treatment position. Usage of a 4D-CT scan during the simulation process was not mandatory.

For patients who had indications for radiotherapy postsurgery, the bronchial stump and specified nodal stations were included in the clinical target volume (CTV) (11), and a margin of 0.5 to $0.8 \mathrm{~cm}$ was added to form the PTV. For 
Table 1 Summary of patient characteristics and clinical information of patients by RP status

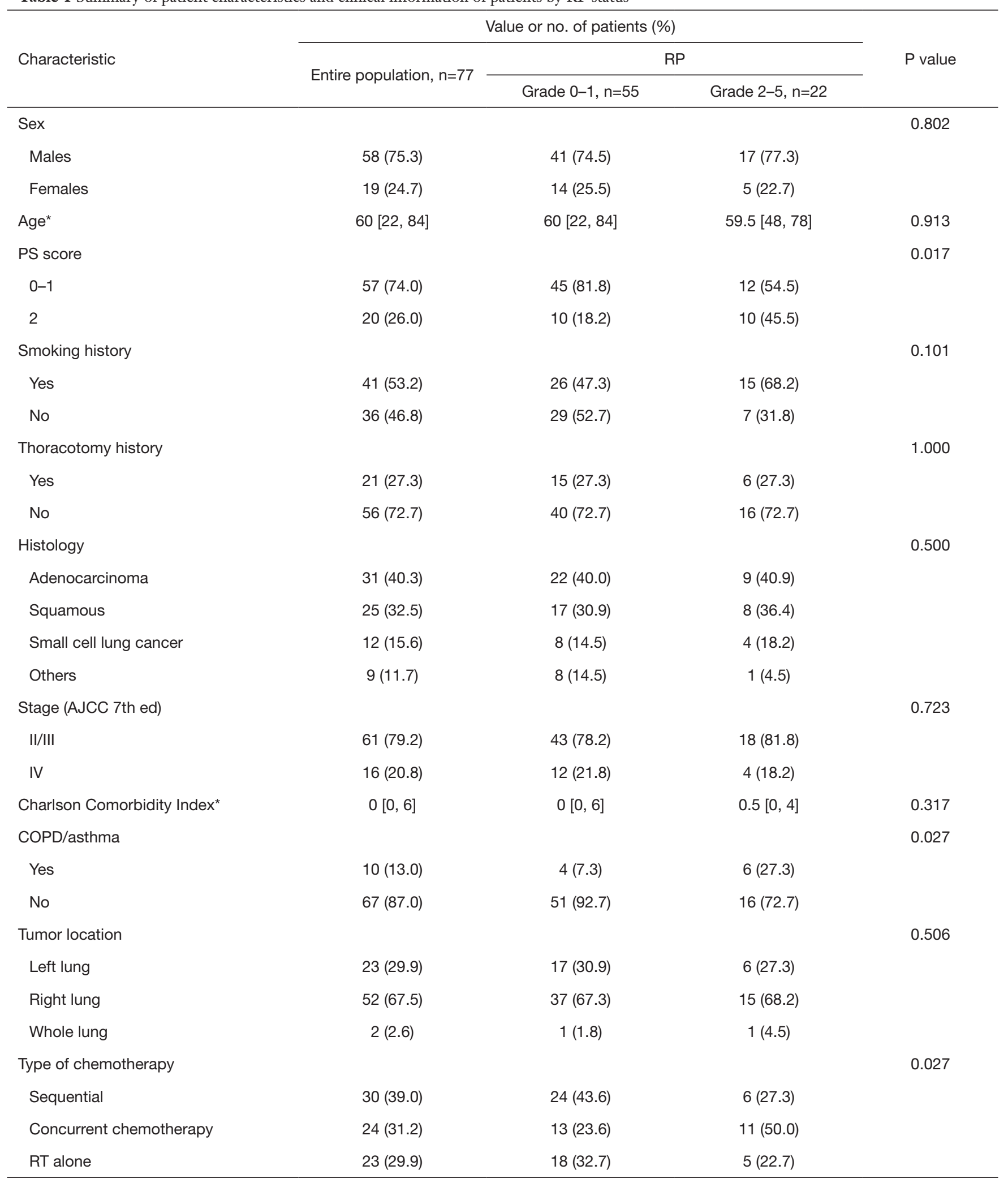

Table 1 (continued) 
Table 1 (continued)

\begin{tabular}{|c|c|c|c|c|}
\hline \multirow{2}{*}{ Characteristic } & \multicolumn{3}{|c|}{ Value or no. of patients (\%) } & \multirow{2}{*}{$P$ value } \\
\hline & Entire population, $n=77$ & Grade $0-1, n=55$ & Grade $2-5, n=22$ & \\
\hline Normal lung volume $\left(\mathrm{cm}^{3}\right)^{\#}$ & $3,112.7 \pm 888.9$ & $3,081.9 \pm 850.7$ & $3,189.9 \pm 995.1$ & 0.633 \\
\hline \multicolumn{5}{|l|}{ Changing level $(\%)^{\star}$} \\
\hline CRP & $287.4(-97.0,5,818.5)$ & $76.7(-97.0,4,944.8)$ & $784.4(-80.5,5,818.5)$ & 0.000 \\
\hline Hemoglobin & $-3.5(-24.8,53.0)$ & $-2.9(-23.1,14.7)$ & $-5.7(-24.8,53.0)$ & 0.931 \\
\hline Lactate dehydrogenase & $-3.8(-56.4,76.5)$ & $-0.5(-43.5,67.5)$ & $-6.5(-56.4,76.5)$ & 0.409 \\
\hline
\end{tabular}

*, median and range; \#, mean \pm SD. RP, radiation pneumonitis; PS, performance status; AJCC, American Joint Committee on Cancer; COPD, chronic obstructive pulmonary disease; RT, radiation therapy; CRP, C-reactive protein.

Table 2 Summary of dosimetric information of patients by RP status

\begin{tabular}{|c|c|c|c|c|}
\hline \multirow{3}{*}{ Characteristic } & \multicolumn{3}{|c|}{ Value or no. of patients (\%) } & \multirow{3}{*}{$P$ value } \\
\hline & \multirow{2}{*}{ Entire population, $\mathrm{n}=77$} & \multicolumn{2}{|c|}{$\mathrm{RP}$} & \\
\hline & & Grade $0-1, n=55$ & Grade $2-5, n=22$ & \\
\hline VMAT plans & & & & 0.247 \\
\hline Single arc & $10(13.0)$ & $6(10.9)$ & $4(18.2)$ & \\
\hline Triple arcs & $5(6.5)$ & $4(7.3)$ & $1(4.5)$ & \\
\hline PTV volume $\left(\mathrm{cm}^{3}\right)^{\#}$ & $423.7 \pm 260.9$ & $394.3 \pm 237.6$ & $497.3 \pm 305.6$ & 0.118 \\
\hline Radiation dose to PTV (Gy)* & $56.0(45.0,64.0)$ & $54.0(45.0,64.0)$ & $60.0(45.0,64.0)$ & 0.086 \\
\hline Delivery time (s) & $187.8 \pm 42.1$ & $188.7 \pm 42.2$ & $185.5 \pm 42.8$ & 0.760 \\
\hline \multicolumn{5}{|l|}{ Lung ${ }^{\#}$} \\
\hline $\mathrm{V}_{10 G \mathrm{G}}(\%)$ & $30.0 \pm 7.1$ & $28.4 \pm 7.2$ & $33.7 \pm 5.1$ & 0.003 \\
\hline $\mathrm{V}_{20 \mathrm{~Gy}}(\%)$ & $20.9 \pm 5.7$ & $20.0 \pm 6.2$ & $23.1 \pm 3.6$ & 0.028 \\
\hline MLD (Gy) & $11.5 \pm 2.8$ & $11.1 \pm 2.9$ & $12.6 \pm 2.2$ & 0.032 \\
\hline
\end{tabular}

*, median and range; \#, mean \pm SD. Abbreviations: RP, radiation pneumonitis; VMAT, volumetric modulated arc therapy; PTV, planning target volume; $\mathrm{Vn}$, volume of lung receiving at least $\mathrm{n}$ Gy of radiation dose; MLD, mean lung dose; MHD, mean heart dose.

locally/advanced patients who were candidates for thoracic radiation therapy, the gross tumor volume (GTV) was defined as the visualization of any gross tumor (GTV-P) and lymph nodes involved (GTV-N). If present, atelectasis was not included in the GTV and was facilitated by a PET/CT fusion to the planning CT. Elective node irradiation was omitted. With regard to the margin that should be added from GTV to PTV to cover the subclinical invasion and trade off the setup error, a 1.0 - to $1.5-\mathrm{cm}$ margin and a 0.5 - to $0.8-\mathrm{cm}$ margin were placed around the GTV-P and GTV-N, respectively. The OARs delineated included double lungs, spinal cord, esophagus, and heart. No margins were added to the OAR. Furthermore, a $\mathrm{kV}$-cone beam CT (CBCT) procedure was performed once a week during radiotherapy treatment to verify the correct patient position and target motion. 
All VMAT plans were generated using single to triple rotation arcs. These plans were delivered by the Elekta Synergy linear accelerator with 6 or $10 \mathrm{MV}$ photons. Ninety-five percent of the PTVs received $100 \%$ of the prescribed dose, and the maximum dose of the PTV should not have exceeded $107 \%$ of the prescription dose. The maximum dose to the spinal cord was $45 \mathrm{~Gy}$, and the dose constraint for the heart was mean heart dose (MHD) $\leq 30$ Gy. For lungs, the V20 and V5 were limited to $30 \%$ and $50 \%$, respectively, and the mean lung dose (MLD) was $\leq 17 \mathrm{~Gy}$.

\section{Follow-up evaluations}

Patient evaluations took place weekly during radiation, once a month until 6 months after RT and then at 3 to 6 months intervals thereafter, with interval history and physical examination, along with basic laboratory studies and CT scans. Patients were evaluated strictly according to the standards mentioned above. A diagnosis of RP, acute radiation esophagitis was made by a consensus of at least two radiation oncologists based on the clinical symptoms, with or without radiographic infiltrate changes. Grading was conducted according to the Common Terminology Criteria for Adverse Events (CTCEA) version 4.0. The grade of pneumonitis was defined as follows: grade 1 (asymptomatic or requiring no treatment), grade 2 [symptomatic, requiring medical intervention or limiting instrumental active daily life (ADL)], grade 3 (severely symptomatic, limiting self care and ADL, or oxygen indicated), grade 4 [life-threatening, respiratory compromise, urgent intervention indicated (e.g., tracheostomy or intubation)], and grade 5 (death). Progression-free survival (PFS) was defined as the time from the first day of radiotherapy to the date of progression or death (whichever occurred first). Overall survival (OS) was the time from radiotherapy until death from any cause.

\section{Data evaluation}

Patients were grouped on the basis of the presence of grades 0 to 1 (asymptomatic) vs. grades 2 to 5 (symptomatic) RP. Changing levels of laboratory results were defined by the formulation of [(Result B) minus (Results A)]/(Result A). In the statistical analysis, descriptive statistics were calculated for discrete variables and continuous variables. Clinical factors, changing levels of laboratory test results and dosimetric parameters were evaluated using univariate analysis for estimating the correlation with the symptomatic
$\mathrm{RP}$. The independent samples $t$-test or the $\chi 2$-test were used for univariate analysis. Multivariate analysis was performed using the logistic regression model (backward: LR) containing all of the variables that attained or trended toward univariate statistical significance $(\mathrm{P} \leq 0.05)$ (Tables 1,2). The Kaplan-Meier method was used to generate the actuarial OS and PFS. The results were considered statistically significant when the $\mathrm{P}$-value was $<0.05$. Statistical tests were based on a 2-sided significance level. All data analyses were performed with SPSS version 19.0 software (SPSS Inc., Chicago, Illinois, USA).

\section{Results}

\section{Acute toxicity and initial outcome results}

After a median follow-up period of 14.8 months (range, 2.3 to 42.4 months), 30 patients developed RP after thoracic radiotherapy. In total, 8 patients $(10.4 \%)$ were grade 1 (G1), $13(16.9 \%)$ grade 2,7 (9.1\%) grade 3, and 2 (2.6\%) G5. No patient had G4 RP. The overall incidence of symptomatic RP (Grade $\geq 2$ by CTCAE) was $28.6 \%$ in the entire cohort. A total of 27 patients developed RP 1 to 3 months after treatment completion, and 3 patients developed asymptomatic RP 4 to 6 months after radiation (126, 141 and 165 days, respectively). Symptomatic RP was diagnosed at a median time of 39 days after treatment completion (range, 22 to 88 days). For the 2 patients who developed fatal RP, the time from treatment completion to onset of was 29 days and 45 days. Both patients developed a lowgrade fever, a dry cough, shortness of breath and chest pain during the course of disease and succumbed to respiratory failure within one month, although timely, active treatment was administered for inflammatory lung disease. The remaining patients with symptomatic RP all recovered after supportive and symptomatic therapies including oral or intravenous steroids, oxygen, and antibiotics.

In addition, 22 patients experienced acute radiation esophagitis. In total, 8 patients (10.4\%) were G1, 10 (13.0\%) G2, and 4 (5.2\%) were G3. Compared with the exclusive radiotherapy group, hematologic toxicity was increased in the chemoradiation group, but no acute toxicity > G3 or interruptions were noted. The median PFS and OS were 7.2 and 14.8 months, respectively.

\section{Potential factors predicting symptomatic $R P$}

Clinical information and dosimetric parameters were 
Table 3 Multivariate logistic regression analysis of potential factors predicting symptomatic RP

\begin{tabular}{lcccc}
\hline Parameter & Sig. & Exp (B) & \multicolumn{2}{c}{$95 \%$ Cl of EXP (B) } \\
\cline { 3 - 4 } $\mathrm{V}_{10 \mathrm{~Gy}}[\%]$ & & 1.138 & Lower limit Upper limit & 1.021 \\
CRP changing level [\%] & 0.019 & 1.048 & 1.010 & 1.088 \\
Constant & 0.013 & 0.004 & - \\
\hline
\end{tabular}

RP, acute radiation pneumonitis; PS, performance status; Vn, volume of lung receiving at least $n$ Gy of radiation dose; CRP, C-reactive protein.

analyzed under univariate analysis as candidate factors predicting symptomatic RP (Tables 1 and 2). Among clinical information, a poorer PS score $(=2)$ was a strong predictor of symptomatic RP $(\mathrm{P}=0.017)$. For the 10 patients who had a history of pulmonary disease, the incidence of symptomatic RP was $60.0 \%$ compared with $23.9 \%$ among the patients without a history of pulmonary disease $(\mathrm{P}=0.027)$. In addition, a trend of increased risk of symptomatic RP was noted in the patients treated with concurrent chemoradiation compared with patients treated with sequential treatment or radiotherapy alone $(45.8 \%$ vs. $20.8 \%, \mathrm{P}=0.027)$. Of interest, the change in CRP level was the only variable that was highly significant based on the independent samples $t$-test $(\mathrm{P}=0.000)$. No other clinical variables such as sex, age, smoking history, thoracotomy history, histology, stage, Charlson comorbidity index, tumor location, and normal lung volume, were significant. We also analyzed a number of dosimetric variables, such as PTV volume, radiation dose, $\mathrm{V}_{5}, \mathrm{~V}_{10}, \mathrm{~V}_{20}, \mathrm{MLD}$ and delivery time. MLD, $V_{5}, V_{10}$ and $V_{20}$ were all statistically significant based on $t$-test $(\mathrm{P}=0.032,0.014,0.003$ and 0.028 , respectively).

Only 22 symptomatic RP events, $\mathrm{V}_{10}$ and CRP changing level, were chosen for multivariable analysis as they seemed to be the most statistically significant factors in univariate analysis $(\mathrm{P}=0.003$ and 0.000 , respectively). However, based on the multivariable analysis, $\mathrm{V}_{10}$ (OR: $1.138,95 \% \mathrm{CI}$, 1.021-1.268, $\mathrm{P}=0.019)$ and the $\mathrm{CRP}$ changing level (OR: $1.048,95 \%$ CI, $1.010-1.088, \mathrm{P}=0.013)$, they were still meaningful in the final multivariable model (Table 3).

\section{Dose parameter benefit of VMAT}

For PTV the following data were reported: $\mathrm{D}_{1 \%}=106.4 \% \pm 2.3 \%$, $\mathrm{D}_{99 \%}=90.9 \% \pm 5.6 \%, \mathrm{~V}_{95 \%}=96.8 \% \pm 3.1 \%$, and $\mathrm{D}_{5 \%}-\mathrm{D}_{95 \%}$ $=9.9 \% \pm 2.0 \%$. Dose homogeneity data revealed that VMAT obtained the planning objectives for most of the parameters considered, especially the target coverage and dose homogeneity (PTV $\left.V_{95 \%}=96.8 \% \pm 3.1 \%\right)$. In terms of organs at risk, VMAT obtained better sparing of normal tissue: heart irradiation was not of major concern as the $\mathrm{V}_{5}$ and $\mathrm{V}_{30}$ of heart for VMAT were $43.2 \% \pm 29.9 \%$ and $14.1 \% \pm 12.6 \%$; the esophagus volume irradiated to doses higher than 60 Gy was $8.1 \% \pm 12.9 \%$. The maximum dose of Spinal Cord was 34.4 \pm 9.5 Gy.

\section{Discussion}

In thoracic radiotherapy, the most commonly observed dose-limiting toxicity is RP. To date, only a few studies have reported the RP incidence in lung cancer patients treated with VMAT. A retrospective review of the MD Anderson, showed that the overall incidence of symptomatic RP [Radiation Therapy Oncology Group (RTOG) Grades 2-5] in lung cancer cases treated by IMRT was $37 \%$, and the rates of grade $\geq 3 \mathrm{RP}$ were $11 \%$ at 6 months and $14 \%$ at 12 months (12). In a study analyzing the toxicity associated with 3D-CRT scans done on 222 patients and IMRT scans done on 68 patients, the rate of grade $\geq 3 \mathrm{RP}$ was only $8 \%$ in the IMRT group but $32 \%$ in the $3 \mathrm{D}$-CRT group (13). In this study, the overall incidence of symptomatic RP (CTCAE grades 2 to 5 ) was $28.6 \%$ in the entire group, and the rate of grade $\geq 3$ treatment-related pneumonitis was $11.7 \%$. The RP incidence is similar between our study and existing literature on the lung cancer patients treated with IMRT. With an acceptable incidence rate of RP, it suggests that VMAT is a safe and clinically feasible treatment modality for lung cancer.

Many studies have also demonstrated the correlation between dose volume histogram data and the risk of RP in lung cancer (14-16). The most appropriate published RP related dosimetric factors include $M L D, V_{20}$ and $V_{30}(17,18)$. Graham et al. reported the risk of $\geq$ Grade 2 pneumonitis in 99 NSCLC patients who were treated with a 3D-CRT scan at the Washington University Medical Center, and 
$\mathrm{V}_{20}$ was the only independent predictor of grade 2 or greater pneumonitis according to multivariate analysis (18). Rodrigues et al. performed a systematic review to assess the predictive ability of various dose-volume histogram parameters, MLD and normal tissue complication probability in the incidence of RP (19). Most studies did show an association between the RP risk and the percentage of lung volume receiving greater than or equal to $20 \mathrm{~Gy}$, and there were few data that focused on lower doses. However, unlike the 3D-CRT and IMRT scans, the lung volume receiving $10 \mathrm{~Gy}$, instead of $M L D, V_{5}$ and $V_{20}$, remained a significant factor of predicting $\mathrm{RP}$ in the final multivariable analysis, which indicated that the volume of lung receiving lower doses of radiation, especially $V_{10}$, might be an important factor in RP when VMAT is used. The results may differ for a few reasons. One possible reason is the favorable tumor coverage with VMAT compared with 3D-CRT/IMRT, in which the volumes were exposed to high doses decrease. The volume of lung that receives lower doses subsequently becomes larger. Another possible reason involves the different evaluation criteria, including CTCAE, RTOG and the World Health Organization (WHO) clinical scoring criteria, which were used in above studies, possibly affecting the consistency of evaluation. In addition, different statistical classification methods may also have contributed to the results. Patients were divided into grades 0 to $1 v s$. grades 2 to $5 \mathrm{RP}$ in the present study, whereas some studies focused on the patients with a diagnosis of grade $\geq 3$ RP. According to our study, efforts to minimize $\mathrm{V}_{10}$ are important in the VMAT planning, although further research is needed to validate these findings.

With the idea that increasing the radiation dosage would be able to improve both local-regional control and OS, RTOG 0617 was designed to address the issues of whether or not high doses offer better outcomes when compared with the standard dosage of thoracic radiation therapy in stage III NSCLC patients based on the 3D-CRT/IMRT scans (20). The results showed that high dose radiation was not better than a standard dosage of radiation therapy and might be potentially harmful. Among other factors included in the multivariate analyses, increasing values of heart $V_{5}$ and heart $\mathrm{V}_{30}$ in the high dose group might best explain why patients administered a high dosage of radiation did worse than the patients who were administered a standard dosage of radiation. Several studies demonstrated that VMAT could reduce delivery time and MUs compared with IMRT (21-23). Reduction in delivery time has the potential of decreasing the uncertainty occurred during treatment.
In addition, VMAT allows the simultaneous variation of gantry rotation speed, leaf translation speed and dose rate during treatment delivery (24), which may provide more conformal target coverage. In the current study, the $\mathrm{V}_{5}$ and $\mathrm{V}_{30}$ of the heart for VMAT were reduced when compared with the IMRT scan for the RTOG 0617 group $(43.2 \%$ vs. $50.4 \% ; 14.1 \%$ vs. $20.0 \%$ ). The $\mathrm{V}_{60}$ of esophagus was also reduced $(8.1 \%$ vs. $13.8 \%)$. These results demonstrated that better sparing of normal tissue and delivery efficiency were obtained with VMAT.

$\mathrm{RP}$ is an inflammatory reaction to ionizing radiation (25), which is supported by numerous studies that have linked $\mathrm{RP}$ with higher levels of systemic pro-inflammatory cytokines and bronchoalveolar immune cells $(26,27)$. Thus, inflammatory factors may have some predictive value for the occurrence of RP. Elevated CRP levels or white blood cell counts were reported during acute exacerbation of idiopathic pulmonary fibrosis (28). These features correspond with the results of our study in which elevated CRP levels during radiation therapy were associated with the risk of symptomatic $\mathrm{RP}(\mathrm{OR}=1.065, \mathrm{P}=0.003)$. Therefore, if a patient has a greater increase in CRP levels during radiotherapy, special consideration is needed when applying subsequent radiotherapy and frequent reexaminations should be given to avoid severe post-RP.

\section{Limitation}

The limitations of the current analysis are its heterogeneity of the population and retrospective design. However, as the focus of the study is on RP, instead of survival, and $V_{10}$ is a dosimetric parameter based on DVH graph, there was little effect of heterogeneity in disease stage and radiation dose on the results. Therefore, the data remained credible and valuable as clinical and dosimetric parameters were still comparable among heterogenous populations. As a retrospective study, the determination of RP can be subjective and challenging. Moreover, the minimum followup period was too short for pneumonitis assessment. However, as the patients with short follow-up time (less than 6 months) did not suffer from RP after their follow-up completion, we believe the shortened follow-up time had a limited effect on the outcomes.

In addition, low incidence of RP may be associated with the low proportion of smokers in our group. However, as a large number of patients $(31 / 77)$ presented with adenocarcinoma and inclusion criteria did not involve smoking status, the low proportion smokers in our group is 
not surprising. Of course, more attention needs to be paid to this issue in future studies.

All of these features are inherent to the study. However, the data was reliable with a strict selection of patients, consistent evaluation of the data and regular follow-ups in this single center study. A further prospective study would be helpful to better understand the RP in lung cancer patients treated with VMAT.

\section{Conclusions}

As the incidence rate of RP was acceptable in our study, we believe that VMAT is a viable option for thoracic radiotherapy of lung cancer. However, we found that patients with a higher $V_{10}$ should be carefully evaluated in a VMAT plan. Unlike the previous study, the lung volume receiving $10 \mathrm{~Gy}$, instead of $\mathrm{V}_{5}$, remained a significant factor for predicting ARP in our group. Efforts to minimize $\mathrm{V}_{10}$ are important in the VMAT planning. Finally, for patients with greater increases in the CRP level, the VMAT technique should be administered with caution. Further research is needed to validate these findings.

\section{Acknowledgements}

We thank Dr. Shi-Rong Zhang and Dr. Yao Ren and for their statistical assistance, and we thank Dr. Qing-Hua Deng for his helpful suggestions in data collection.

Funding: This work was supported by Hangzhou Science and Technology Bureau (grant number 20150633B42) and Department of Health of Zhejiang Province (grant number 2017KY534) in the design of the study and interpretation of data.

\section{Footnote}

Conflicts of Interest: The authors have no conflicts of interest to declare.

Ethical Statement: The study was approved by the Institutional Ethics Committee of Medical Ethics Committee of Hangzhou Cancer Hospital (No. HZCH-2016-18).

\section{References}

1. Tsoutsou PG, Koukourakis MI. Radiation pneumonitis and fibrosis: mechanisms underlying its pathogenesis and implications for future research. Int $\mathrm{J}$ Radiat Oncol Biol
Phys 2006;66:1281-93.

2. Palma DA, Senan $S$, Tsujino K, et al. Predicting radiation pneumonitis after chemoradiation therapy for lung cancer: an international individual patient data meta-analysis. Int $\mathrm{J}$ Radiat Oncol Biol Phys 2013;85:444-50.

3. Okubo M, Itonaga T, Saito T, et al. Predicting risk factors for radiation pneumonitis after stereotactic body radiation therapy for primary or metastatic lung tumours. $\mathrm{Br} \mathrm{J}$ Radiol 2017;90:20160508.

4. Guo M, Huang E, Liu X, et al. Volumetric Modulated Arc Therapy versus Fixed-Field Intensity-Modulated Radiotherapy in Radical Irradiation for Cervical Cancer without Lymphadenectasis: Dosimetric and Clinical Results. Oncol Res Treat 2018;41:105-9.

5. Holt A, Van Gestel D, Arends MP, et al. Multi-institutional comparison of volumetric modulated arc therapy vs. intensity-modulated radiation therapy for head-and-neck cancer: a planning study. Radiat Oncol 2013;8:26.

6. Shrimali RK, Arunsingh M, Reddy GD, et al. Actual gains in dosimetry and treatment delivery efficiency from volumetric modulated arc radiotherapy for inoperable, locally advanced lung cancer over five-field forwardplanned intensity-modulated radiotherapy. Indian J Cancer 2017;54:155-60.

7. Jiang X, Li T, Liu Y, et al. Planning analysis for locally advanced lung cancer: dosimetric and efficiency comparisons between intensity-modulated radiotherapy (IMRT), singlearc/partial-arc volumetric modulated arc therapy (SA/PAVMAT). Radiat Oncol 2011;6:140.

8. Wijsman R, Dankers F, Troost EG, et al. Comparison of toxicity and outcome in advanced stage non-small cell lung cancer patients treated with intensity-modulated (chemo-) radiotherapy using IMRT or VMAT. Radiother Oncol 2017;122:295-9.

9. Kumar G, Rawat S, Puri A, et al. Analysis of dose-volume parameters predicting radiation pneumonitis in patients with esophageal cancer treated with 3D-conformal radiation therapy or IMRT. Jpn J Radiol 2012;30:18-24.

10. Vogelius IS, Westerly DC, Cannon GM, et al. Intensitymodulated radiotherapy might increase pneumonitis risk relative to three-dimensional conformal radiotherapy in patients receiving combined chemotherapy and radiotherapy: a modeling study of dose dumping. Int J Radiat Oncol Biol Phys 2011;80:893-9.

11. Feng W, Fu XL, Cai XW, et al. Patterns of local-regional failure in completely resected stage IIIA(N2) non-small cell lung cancer cases: implications for postoperative radiation therapy clinical target volume design. Int J Radiat 
Oncol Biol Phys 2014;88:1100-7.

12. Jiang ZQ, Yang K, Komaki R, et al. Long-term clinical outcome of intensity-modulated radiotherapy for inoperable non-small-cell lung cancer: the MD Anderson experience. Int J Radiat Oncol Biol Phys 2012;83:332-9.

13. Yom SS, Liao Z, Liu HH, et al. Initial evaluation of treatment-related pneumonitis in advanced-stage nonsmall-cell lung cancer patients treated with concurrent chemotherapy and intensity-modulated radiotherapy. Int J Radiat Oncol Biol Phys 2007;68:94-102.

14. Takahashi S, Go T, Kasai Y, et al. Relationship between dose-volume parameters and pulmonary complications after neoadjuvant chemoradiotherapy followed by surgery for lung cancer. Strahlenther Onkol 2016;192:658-67.

15. Schallenkamp JM, Miller RC, Brinkmann DH, et al. Incidence of radiation pneumonitis after thoracic irradiation: dose-volume correlates. Int J Radiat Oncol Biol Phys 2007;67:410-6.

16. Tsujino K, Hashimoto T, Shimada T, et al. Combined analysis of V20, VS5, pulmonary fibrosis score on baseline computed tomography, and patient age improves prediction of severe radiation pneumonitis after concurrent chemoradiotherapy for locally advanced non-small-cell lung cancer. J Thorac Oncol 2014;9:983-90.

17. Fay M, Tan A, Fisher R, et al. Dose-volume histogram analysis as predictor of radiation pneumonitis in primary lung cancer patients treated with radiotherapy. Int J Radiat Oncol Biol Phys 2005;61:1355-63.

18. Graham MV, Purdy JA, Emami B, et al. Clinical dosevolume histogram analysis for pneumonitis after $3 \mathrm{D}$ treatment for non-small cell lung cancer (NSCLC). Int J Radiat Oncol Biol Phys 1999;45:323-9.

19. Rodrigues G, Lock M, D'Souza D, et al. Prediction of radiation pneumonitis by dose-volume histogram parameters in lung cancer: a systematic review. Radiother Oncol 2004;71:127-38.

20. Bradley JD, Paulus R, Komaki R, et al. Standard-dose

Cite this article as: $\mathrm{Wu} \mathrm{K}, \mathrm{Xu} \mathrm{X}, \mathrm{Li} \mathrm{X}$, Wang J, Zhu L, Chen X, Wang B, Zhang M, Xia B, Ma S. Radiation pneumonitis in lung cancer treated with volumetric modulated arc therapy. J Thorac Dis 2018;10(12):6531-6539. doi: 10.21037/jtd.2018.11.132 versus high-dose conformal radiotherapy with concurrent and consolidation carboplatin plus paclitaxel with or without cetuximab for patients with stage IIIA or IIIB non-smallcell lung cancer (RTOG 0617): a randomised, two-by-two factorial phase 3 study. Lancet Oncol 2015;16:187-99.

21. Nguyen K, Cummings D, Lanza VC, et al. A dosimetric comparative study: volumetric modulated arc therapy vs intensity-modulated radiation therapy in the treatment of nasal cavity carcinomas. Med Dosim 2013;38:225-32.

22. Taggar AS, Graham D, Kurien E, et al. Volumetricmodulated arc therapy versus intensity-modulated radiotherapy for large volume retroperitoneal sarcomas: A comparative analysis of dosimetric and treatment delivery parameters. J Appl Clin Med Phys 2018;19:276-81.

23. Yoo $\mathrm{S}, \mathrm{Wu}$ QJ, Lee WR, et al. Radiotherapy treatment plans with RapidArc for prostate cancer involving seminal vesicles and lymph nodes. Int J Radiat Oncol Biol Phys 2010;76:935-42.

24. Otto K. Volumetric modulated arc therapy: IMRT in a single gantry arc. Med Phys 2008;35:310-7.

25. Roberts CM, Foulcher E, Zaunders JJ, et al. Radiation pneumonitis: A possible lymphocyte-mediated hypersensitivity reaction. Ann Intern Med 1993;118:696-700.

26. Stenmark MH, Cai XW, Shedden K, et al. Combining physical and biologic parameters to predict radiationinduced lung toxicity in patients with non-small-cell lung cancer treated with definitive radiation therapy. Int J Radiat Oncol Biol Phys 2012;84:e217-22.

27. Nakayama Y, Makino S, Fukuda Y, et al. Activation of lavage lymphocytes in lung injuries caused by radiotherapy for lung cancer. Int J Radiat Oncol Biol Phys 1996;34:459-67.

28. Sanuki N, Ono A, Komatsu E, et al. Association of computed tomography-detected pulmonary interstitial changes with severe radiation pneumonitis for patients treated with thoracic radiotherapy. J Radiat Res 2012;53:110-6. 